\title{
Optimization of HiPIMS Photocatalytic Titania Coatings for Low Temperature Deposition
}

\author{
M Ratova $^{1}$, GT West ${ }^{1}$, PJ Kelly ${ }^{1}$ \\ ${ }^{1}$ Surface Engineering Group, Manchester Metropolitan University, Manchester M1 5GD
}

Abstract

Titanium dioxide in its anatase form is widely used in photocatalytic applications due to its high photocatalytic activity, stability and low cost. Titania coatings directly deposited by conventional magnetron sputtering tend to have an amorphous microstructure. For the anatase structure to develop, substrate heating or post-deposition thermal treatment is usually required, with the anatase crystal phase generally forming at temperatures in excess of $400{ }^{\circ} \mathrm{C}$. This precludes the choice of thermally sensitive substrate materials for the photoactive coating.

Depending on the nature of the driving voltage waveform, high power impulse magnetron sputtering (HiPIMS) has been shown to deliver a relatively low thermal flux to the substrate, whilst still allowing the direct deposition of crystalline titania coatings.

Consequently, this technique offers the potential to deposit photocatalytically active titania coatings directly onto polymeric substrates and, therefore, open up a range of new applications. In the present work a range of titanium dioxide thin films were deposited by HiPIMS onto glass substrates in order to study the influence of various process parameters, such as pressure, pulse frequency and pulse duration on coating structure and photocatalytic properties. The photocatalytic properties of the coatings were assessed by their ability to degrade the organic dye methylene blue under UV and fluorescent light irradiation. The degradation rate of methylene blue was calculated by measuring its absorption peak height at $665 \mathrm{~nm}$ in continuous mode under UV / fluorescent light source. The hydrophilic properties of the coatings were also investigated by measuring the contact angle of water droplets on the coating surfaces. Experimentally, the optimum conditions to maximise the photocatalytic 
performance of the coatings were found. The influence of various deposition parameters on the photocatalytic properties and crystal structure of the coatings is discussed.

Optimised coatings then were deposited onto polymeric substrates, such as polyethylene terephthalate (PET) and polycarbonate, to assess the suitability of using this method for highenergy, low-temperature deposition of photoactive titania coatings and the relevance of the optimised condition were tested for other types substrates than glass. It was found that titania coatings deposited by HiPIMS directly onto polymeric substrates showed relatively high levels of activity in their as-deposited state.

The ability to deposit crystalline titania with photocatalytic functionality at temperatures low enough to enable the use of polymer substrates is a significant advance in the field. It could potentially allow the production of high volumes of photocatalytic material on substrates, such as polymer web; which is not possible with current deposition techniques. 


\section{Introduction}

Photocatalytic titanium dioxide (or titania) films are extensively studied due to their efficiency in the degradation of organic compounds. They find many applications, such as the purification of air and water [1,2], various types of self-cleaning materials [3], antimicrobial coatings [4], etc. Due to the expanding application areas of photocatalytic titania coatings, there is a growing need for extending the range of substrates that coatings can be deposited onto. Polymeric materials used as substrates for photocatalytic films offer such advantages as flexibility, low cost, easy fabrication and customisation of the substrate properties depending on the type of polymer used. Several methods have been successfully applied for the deposition of photocatalytic titania coatings onto polymeric substrates, such as dip-coating [5], atomic layer deposition (ALD) [6], sol-gel [7], etc.

Magnetron sputtering is a widely studied and convenient way of depositing photocatalytic titania coatings. However, there are some limitations for use of this technique on polymeric substrates. The coatings deposited by conventional magnetron sputtering tend to have an amorphous microstructure [8-10]and thus very low photocatalytic activity (the deposition of crystalline titania coatings by magnetron sputtering is possible by using RF power supplies or substrate bias, however these methods will result in significant substrate heating and are therefore not discussed here [11]). For crystalline structures to develop, post-deposition thermal treatment is usually required, with the anatase crystal phase generally forming at temperatures in excess of $400{ }^{\circ} \mathrm{C}$. This significantly limits the choice of materials that may be used as a substrate for photocatalytic coatings.

High power impulse magnetron sputtering (HiPIMS) is a relatively new technology in magnetron sputtering, introduced by Kouznetsov et al in 1999 [12]. It utilises high peak voltages and very high peak currents at low durations, which results in similar average power, but much lower duties, compared to pulsed DC magnetron sputtering. HiPIMS is reported to increase the ionisation fraction of the coating flux - up to $70 \%$ for titanium targets [13] (less 
than $1 \%$ for DC magnetron sputtering), and thus enhances the film structure and makes possible the deposition of crystalline thin films without additional heat treatment. A dense plasma with a high degree of ionisation allows control of the energy and direction of the sputtering flux [14]. The thermal energy flux delivered to the substrate in HiPIMS deposition is reported to be several times lower than for DC or pulsed DC magnetron sputtering at the same time-averaged power [13]. Thus, HiPIMS provides an opportunity to deposit dense crystalline coatings that do not require any further thermal treatment, virtually, on any kind of substrate.

The suitability of the HiPIMS method for the deposition of photoactive titania coatings onto polymeric substrate has already been shown earlier [15]. Several attempts had been made to investigate HiPIMS deposition parameters and their effect on coating properties. Thus, Reed et al. [14] studied the influence of pressure on the growth of titanium and hafnium films; films deposited at higher working pressures were characterised with void-free surfaces and growth of crystals with one preferential orientation. Nouvellon et al. published a work on the influence of peak current values on titanium dioxide films [16], the presence of rutile phase was reported in titania films deposited at high peak currents (400A), while for lower peak currents the crystallinity of the films varied with oxygen flow . Čada et al. studied the effects of working gas pressure and discharge current on thin titania films [17]. However no systematic study on the influence of deposition conditions on photocatalytic properties of the coatings has been carried out to date. Thus, there is an obvious need for a study on HiPIMS deposition parameters in order to maximise the photocatalytic performance of titania coatings.

In the present work, thin titania films were deposited onto glass substrates under varied conditions at the same mean power in order to study the effect of pressure, pulse frequency and pulse width on the structure and photocatalytic properties of the films. Only one parameter was changed at a time, allowing a comprehensive mapping of deposition effects to be obtained. An optimum set of conditions for the deposition of titania coatings was identified 
by analysing such factors as process stability, and the structural and photocatalytic properties of the deposited coatings. Using the optimum set of conditions (the deposition conditions that maximised the resulting photocatalytic performance of the titania coatings), coatings were deposited onto selected types of polymeric substrates, such as polyethylene terephthalate (PET) and polycarbonate (PC), and then re-analysed.

\section{Experimental procedure}

The coatings were deposited in Nordiko sputtering rig with high vacuum achieved (shown on Figure 1) by a combination of turbo-molecular and rotary pumps. The single $300 \mathrm{~mm} \times 100$ mm titanium target (99.99\% purity) was fitted onto a GencoaLtd. magnetron that has thicker insulation compared to regular planar magnetrons to prevent arcing and breakdown. Power to the magnetron was delivered in HiPIMS mode using a Huettinger HMP1/1_P2 power supply. The target waveforms (current, voltage and delivered power) were monitored via a Tektronix digital oscilloscope. An example of target waveforms is presented in Figure 2. The substrate was mounted on rotatable drum-shaped substrate holder. The distance between magnetron and substrate holder was kept as $100 \mathrm{~mm}$. Sputtering was carried out in an argon : oxygen atmosphere of $2: 3$ for all deposition runs (10 sccm of Ar and $15 \mathrm{sccm}$ of $\left.\mathrm{O}_{2}\right)$, which corresponded to the poisoned mode for this system. The thresholds of these variable parameters were chosen to maintain stable plasma discharge conditions and, thereby, control over the deposition process. The working pressure was varied in the range of 0.13 to $0.93 \mathrm{~Pa}$. Pulse frequency $(100-300 \mathrm{~Hz})$ and pulse width $(50-200 \mu \mathrm{s})$ were used as two other process variables. To provide a better understanding of the effect of deposition parameters on the properties of the coatings, only one parameter was varied at a time. Thus, for all depositions runs with varied pressure, the pulse frequency was set at $200 \mathrm{~Hz}$ and pulse width at $100 \mu$ s. Variations of pulse frequency were carried out at $0.33 \mathrm{~Pa}$ pressure and $100 \mu$ s pulse width. Similarly, for the investigation of the effect of pulse width the settings for pressure and frequency were kept at $0.33 \mathrm{~Pa}$ and $200 \mathrm{~Hz}$, respectively. 
The coatings were initially deposited onto soda-lime glass substrates. Coating thickness measurements were obtained by means of surface profilometry. All coatings deposited in this project were in the range of $100 \mathrm{~nm}$. The crystal structure of the coatings was analysed by Raman spectroscopy (RenishawInvia, $514 \mathrm{~nm}$ laser). The surface roughness of the coatings was determined using MicroXAM white light surface profilometry.

The photocatalytic properties of the coatings were assessed using the methylene blue (MB) photodecomposition test. Samples of the same geometrical size $\left(1.5 \times 2.5 \mathrm{~cm}^{2}\right)$ were immersed in $40 \mathrm{ml}$ of aqueous MB solution of concentration $1.5 \mu \mathrm{mol} / 1$ (the concentration was defined experimentally to allow the detection of a photocatalytic response of each coating during a 1 hour experiment). The absorption peak height of MB solution at $664 \mathrm{~nm}$ was continuously monitored for one hour using an Ocean Optics USB 2000+ spectrometer. $2 \times$ 15W 352 nm Sankyo Denki BLB lamps (Sankyo Denki Co, Osaka, Japan) were used as UV light source. The spectra of the of the UV light source used in shown in Figure 3. The integrated power flux to the coating was $4 \mathrm{~mW} / \mathrm{cm}^{2}$. The apparent first order rate constant, $\mathrm{k}_{\mathrm{a}}$, was used as a quantitative characterization of the photocatalytic degradation rate of MB. Values for $k_{a}$ were found from the gradient of the graph of $A_{t} / A_{t}=0$ versus time $\left(A_{t}=0\right.$ and $A t$ are the absorption of methylene blue at $664 \mathrm{~nm}$ at time 0 and time of experiment, respectively). Hydrophilic properties of the coatings were estimated via measurements of contact angles of deionised water droplets on the surface of the coating made with a with Kruss goniometer.

Optimised operating conditions were then used to deposit coatings onto PET and PC substrates. No substrate melting or distortion was observed during the deposition process.

\section{Results:}

\subsection{Coatings structural properties}


Structural properties of the coatings were obtained by means of Raman spectroscopy.

According to these results, all the coatings investigated developed a nanophase crystal structure with characteristic Raman peaks used for identification of the crystal phase type. The Raman spectra obtained corresponded to nanophase titania, widely described in literature [18].

The majority of the Raman spectra observed had well-resolved peaks at $153 \mathrm{~cm}^{-1}$, indicating the presence of anatase in the coatings. Overall, the structure of the coatings was identified as a mixed anatase / rutile phase with broad peaks as a result of the overlapping of characteristic anatase peaks at 144, 397, 516 and $638 \mathrm{~cm}^{-1}$ and rutile peaks at 448 and $609 \mathrm{~cm}^{-1}$.

The strong peak observed on all the Raman spectra at $1100 \mathrm{~cm}^{-1}$ can be attributed to titanium - oxygen - silicon dioxide bond formation [19]. The presence of this peak indicates the existence of a strong interaction between the coatings and the glass substrate.

Due to the broadness of the peaks it is not possible to clearly identify the trends in phase transformations with changes in the sputtering parameters, however some general information can be obtained from the shape of the peaks. Thus, with increasing pressure overlapping anatase / rutile peaks between 580 and $650 \mathrm{~cm}^{-1}$ become better defined and have higher intensity, indicating the enhancement of coating crystallinity [18]. On the contrary, Raman spectra of the coatings prepared at lower working pressure have low-intensity broad peaks, which may be taken as evidence of poor crystal structure. Typical examples of the Raman spectroscopy results obtained for the coatings deposited at varied working pressures are shown in Figure 4.

The influence of pulse frequency and pulse width on the coating structures was not so obvious from the Raman spectra obtained.

\subsection{Morphology of the coatings}


According to the results of white light surface profilometry, all coatings had relatively low values of surface roughness and surface area $(5-11 \mathrm{~nm})$. No significant variations in morphology were observed as result of varying the deposition conditions. The results of these measurements are presented in Table I.

\subsection{Photocatalytic activity of the coatings}

Figure 5 shows the results of the dependence of the first order rate constant, $\mathrm{k}_{\mathrm{a}}$, on the varied sputtering parameters, such as pressure, pulse frequency and pulse width, respectively. It can be seen that working pressure has a significant influence on the photocatalytic activity. There was found to be a strong correlation between these two parameters in this experimental array, with photocatalytic activity reaching a maximum value of $3.3 \times 10^{-5}$ at the maximum pressure used(0.93 Pa).

The effect of pulse frequency on photocatalytic activity was less pronounced; increasing pulse frequency from 100 to $200 \mathrm{~Hz}$ doubled the photoactivity. However further increases in pulse frequency did not intensify the process of MB degradation any further.

Changing pulse width from 50 to $100 \mu$ s did not have noticeable effect on the MB degradation process. However further increases of the pulse width value resulted in a sharp drop in photoactivity. The coating deposited at a pulse width of $200 \mu$ s had no detectable photocatalytic activity at all.

\subsection{Hydrophilicity measurements}

The measurements of coating hydrophilicity as a photoinduced phenomenon were beyond the scope of this project. However, the deionised water contact angles on the surface of the coatings were measured for better understanding of the influence of sputtering parameters. Contact angle measurements generally had similar trends to photocatalytic activity results. Thus, coatings produced at higher working pressure had higher hydrophilicity; with the 
coating deposited at the $0.93 \mathrm{~Pa}$ pressure demonstrating superhydrophilic properties with a contact angle of $9^{\circ}$.

Increasing pulse frequency and pulse width resulted in a slight decrease of contact angle values. The results obtained for water droplet contact angle measurements are presented in Figure 6 and Table I.

\subsection{Deposition of coatings onto polymeric substrates under optimised conditions}

Based on structural properties of the coatings and photocatalytic measurements results, two sets of parameters were selected as optimised conditions for further deposition of coatings onto polymeric substrates.

In terms of pressure, the coatings deposited at $0.93 \mathrm{~Pa}$ showed the highest performance in the MB degradation test and more developed crystal structures, thus for both sets of optimised conditions the working pressure chosen was $0.93 \mathrm{~Pa}$.

In terms of pulse frequency, as no obvious trends could be seen for coating crystallinity, the selection was based on the criterion of photocatalytic performance. The rate constant, $\mathrm{k}_{\mathrm{a}}$, had the highest value for coatings deposited at 200, 250 and $300 \mathrm{~Hz}$, thus frequencies of 200 and $300 \mathrm{~Hz}$ were chosen as two possible options for optimum frequency.

The pulse width effect on photocatalytic properties of the coatings suggested the optimum parameters should be in the range $50-100 \mu$ s, as further increases in pulse width resulted in a sharp drop of photocatalytic performance. Therefore, pulse widths of 50 and $100 \mu$ s were suggested as optimum parameters.

Summarising all testing conditions, the coatings were deposited onto polymer substrates under the following two sets of conditions:

1. Working pressure: $0.93 \mathrm{~Pa}$ 
Pulse frequency: $300 \mathrm{~Hz}$

Pulse width: $50 \mu$;

2. Working pressure: $0.93 \mathrm{~Pa}$

Pulse frequency: $200 \mathrm{~Hz}$

Pulse width: $100 \mu$ s.

PET and polycarbonate were used as the options for polymeric substrate. The coatings were, therefore, deposited under optimised conditions onto PET, polycarbonate and glass. No substrate melting or distortion was observed during the process. The photocatalytic activity of the coatings deposited under these conditions was then tested and compared. The results of the MB decomposition test for the optimised coatings are presented in Figure 7.

As can be seen from these results, conditions 1 allowed a further increase of photocatalytic activity compared to initial array of the coatings on glass. The activity of the coatings produced under conditions 2 remained at the same level as the activity measured previously. The coatings demonstrated very similar levels of photocatalytic activity, regardless the substrate type they were deposited onto.

\section{Discussion}

The data obtained in this work demonstrated that the crystal structure and, thus, photoactivity of thin titania films can be altered by changing deposition parameters when operating in HiPIMS mode. Of the parameters tested here, pressure had the greatest influence on photoactivity. Working pressure is an important parameter in magnetron sputtering and it has been shown that the pressure can be used for the control of structural and photocatalytic properties of the coatings [14]. Increasing the working pressure results in reduced mean free path of ions and atoms in the discharge, and thus longer lifetime of excited species and higher plasma ionisation [20]. Reportedly, increasing the working pressure during the deposition 
process is method to deposit coatings with crystalline rather than amorphous structures [21]. The working pressure is one of the key deposition parameters, which influences the kinetic energy of incident Ti particles. Increasing pressure results in higher energy input to the coatings, and in turn, higher crystallinity of the coatings. As shown earlier, the polycrystalline anatase / rutile titania coatings show better performance in terms of photocatalytic activity than purely anatase coatings, as the rutile plays the key role in separating electrons and holes, thus slowing down their recombination [22]. The preferred anatase / rutile ratio varies depending on the source, for instance the well-known commercially available P25 titanium dioxide consists of $80 \%$ anatase phase and $15 \%$ rutile phase [23]. Although the anatase / rutile ratio is not measured here, the high photocatalytic activity of the coatings deposited at higher pressures may be attributed to their crystal structure being a mix of anatase and rutile phases, as can be seen from the Raman spectra obtained. Better peak definition in the Raman spectra may be taken as evidence of improved crystallinity compared to the coatings deposited at low pressure. Compared to anatase titania coatings investigated in earlier work [10] deposited by pulsed DC magnetron sputtering and annealed at $673 \mathrm{~K}$, the coatings deposited by HiPIMS method under optimised conditions were more efficient in terms of MB degradation by a factor of 2 .

To further investigate the effect of deposition conditions on the structural and photocatalytic properties of the coatings, the discharge currents during the on-pulse were plotted using the data obtained with a digital oscilloscope and are presented as functions of pressure, pulse width and pulse frequency in figures 8,9 and 10, respectively. From Figure 8 is it clear that despite the influence this parameter had on photocatalytic behaviour, changes to the working pressure did not affect the pulse peak current values.

The influence of pulse width on pulse peak current is shown in Figure 9. It is noticeable that increasing the pulse width over $100 \mu$ s caused a sharp drop in pulse peak current. High peak currents result in increased flux of ionised species and improved crystallinity of the coatings. 
Consequently, low pulse peak current decreases the ionised particle flux, and results in deposition of coatings with poor crystal structure. This appears to be confirmed by the results of crystal structure and photocatalytic activity obtained here - the coatings deposited at pulse widths with low peak current values (150 and $200 \mu$ s) had amorphous structures and very low photocatalytic activity.

The effect of pulse frequency on the pulse current is less pronounced, as shown in Figure 10.In fact, changes in pulse frequency did not greatly affect the peak current values $\left(1 \times 10^{-5} \mathrm{~s}^{-1}\right.$ at $100 \mathrm{~Hz}$ to $2 \times 10^{-5} \mathrm{~s}^{-1}$ at $300 \mathrm{~Hz}$ ). However, the variation in pulse frequency did have a moderate effect on the photocatalytic activity value. Increased pulse frequency results in higher thermal load on substrate, but in our case the substrates were able to withstand the range of frequencies used [24].

Deposition of photocatalytic coatings onto plastic has commonly led to problems of partial substrate decomposition during the photocatalytic process [25]. This process leads to weakening the bonds between the substrate and coating, and, thus, to coatings peeling off. Generally, the HiPIMS method is reported to improve adhesion of the coatings to the substrate [26]. In may be confirmed with the fact that all the Raman spectra of the titania coatings deposited during this work demonstrated an intense peak at $1100 \mathrm{~cm}^{-1}$, indicating strong coating-substrate bonding. Moreover, the coatings on PET and PC did not show any sign of peeling off after several immersions in MB, as well as after continuous UV irradiation for several hours. Therefore, we can conclude, that even if the process of photocatalytic polymer degradation occurs, the coating-substrate bonding is strong enough to withstand it without peeling off.

The contact angle measurements were carried out in the normal indoor conditions with fluorescent light bulbs used as a light source. While analysing the data obtained we had to assume the fact that during preparation for the contact angle measurements, the coatings were exposed to fluorescent light irradiation for a few minutes. It is often reported, that even when 12 
photoinduced hydrophilicity is a phenomenon independent on photocatalytic activity[27], photogenerated electron and holes are involved in both cases. Thus, the hydrophilicity observed might be partly attributed to the presence of photogenerated oxygen vacancies on the surface of the coatings, as in the case of coatings deposited at higher working pressures which showed superhydrophilic properties. In the case of coatings deposited with varied pulse frequency and pulse width, the effect of deposition conditions was not as pronounced as in the case of pressure. The small drop in the value of contact angle for the coating deposited at 200 $\mu$ s pulse width with absence of any photocatalytic activity and overall amorphous structure, might be attributed to increased number of surface defects that caused partial adsorption of water droplet on the surface [28].

\section{Conclusion}

This work summarises the effect of several sputtering parameters, such as working pressure, pulse frequency and pulse width, on the crystallinity and photocatalytic properties of titania thin films. According to the results of MB degradation tests, among the parameters investigated, pressure was found to have the major influence on the coatings photocatalytic properties of the coatings. Variation of working pressure allowed us to deposit crystalline titania coatings with a mixture of anatase and rutile phases, that showed high efficiency in MB decomposition. Variations of pulse width and pulse frequency were found to change pulse current characteristics to different degree, and thus also had an impact on film photocatalytic properties, though not as significant as the influence of pressure. Superhydrophilicity was observed for several coatings demonstrating high photocatalytic activity, while for the other coatings, low contact angles can be attributed to high numbers of surface defects and water droplet partial adsorption. 
Based on the effect of the initial sputtering parameters, two sets of optimised conditions were selected and successfully used for deposition of photocatalytic titania coatings onto polymeric substrates, namely PET and PC. These coatings were characterised as having high photocatalytic activity in their as-deposited state ( 2 times greater than pulsed DC coatings which had been post-deposition annealed) and good adhesion to the substrates. No substrate melting or distortion occurred during the deposition process. According to the results of photocatalytic MB decomposition, the choice of substrate did not have significant effect on the photocatalytic properties, as coatings deposited under the same conditions onto glass, PET and PC showed almost identical results in terms of photocatalytic activity.

\section{References:}

[1] Y. Paz, Applied Catalysis B: Environmental, 99 (2010) 448-460. 
[2] A. Vidal, Chemosphere, 36 (1998) 2593-2606.

[3] A. Fujishima, X. Zhang, Comptes Rendus Chimie, 9 (2006) 750-760.

[4] A. Markowska-Szczupak, K. Ulfig, A.W. Morawski, Catalysis Today, 169 (2011) 249-257.

[5] J.-H. Yang, Y.-S. Han, J.-H. Choy, Thin Solid Films, 495 (2006) 266-271.

[6] C.-S. Lee, J. Kim, J.Y. Son, W. Choi, H. Kim, Applied Catalysis B: Environmental, 91 (2009) 628-633.

[7] H. Yaghoubi, N. Taghavinia, E.K. Alamdari, Surface and Coatings Technology, 204 (2010) 1562-1568.

[8] A.A. Onifade, P.J. Kelly, Thin Solid Films, 494 (2006) 8-12.

[9] N. Farahani, P.J. Kelly, G. West, M. Ratova, C. Hill, V. Vishnyakov, Thin Solid Films, 520 (2011) 1464-1469.

[10] M. Ratova, P.J. Kelly, G.T. West, I. Iordanova, Surface and Coatings Technology, 228, Supplement 1 (2013) S544-S549.

[11] R.D. Arnell, P.J. Kelly, J.W. Bradley, Surface and Coatings Technology, 188-189 (2004) 158-163.

[12] V. Kouznetsov, K. Macak, J.M. Schneider, U. Helmersson, I. Petrov, Surface and Coatings Technology, 122 (1999) 290-293.

[13] G. West, P. Kelly, P. Barker, A. Mishra, J. Bradley, Plasma Processes and Polymers, 6 (2009) S543-S547.

[14] A.N. Reed, M.A. Lange, C. Muratore, J.E. Bultman, J.G. Jones, A.A. Voevodin, Surface and Coatings Technology, 206 (2012) 3795-3802.

[15] P.J. Kelly, P.M. Barker, S. Ostovarpour, M. Ratova, G.T. West, I. Iordanova, J.W. Bradley, Vacuum, 86 (2012) 1880-1882.

[16] C. Nouvellon, M. Michiels, J.P. Dauchot, C. Archambeau, F. Laffineur, E. Silberberg, S. Delvaux, R. Cloots, S. Konstantinidis, R. Snyders, Surface and Coatings Technology, 206 (2012) 3542-3549.

[17] M. Čada, Z. Hubička, P. Adámek, J. Klusoň, L. Jastrabík, Surface and Coatings Technology, 205, Supplement 2 (2011) S317-S321.

[18] W. Ma, Z. Lu, M. Zhang, Applied Physics A, 66 (1998) 621-627.

[19] F.-M. Liu, T.-M. Wang, Applied Surface Science, 195 (2002) 284-290.

[20] A. Hecimovic, K. Burcalova, A.P. Ehiasarian, Journal of Physics D: Applied Physics, 41 (2008) 095203.

[21] C. Zhang, W. Ding, H. Wang, W. Chai, D. Ju, Journal of Environmental Sciences, 21 (2009) 741-744. 
[22] R.I. Bickley, T. Gonzalez-Carreno, J.S. Lees, L. Palmisano, R.J.D. Tilley, Journal of Solid State Chemistry, 92 (1991) 178-190.

[23] R. Su, R. Bechstein, L. Sø, R.T. Vang, M. Sillassen, B. Esbjörnsson, A. Palmqvist, F. Besenbacher, The Journal of Physical Chemistry C, 115 (2011) 24287-24292.

[24] G.T. West, P.M. Barker, P.J. Kelly, J.W. Bradley, in: 51st Annual Technical Conference Proceedings, Chicago, IL, 2008, pp. 277-281.

[25] J. Jensen, M. Mikkelsen, F.C. Krebs, Solar Energy Materials and Solar Cells, 95 (2011) 2949-2958.

[26] U. Helmersson, M. Lattemann, J. Bohlmark, A.P. Ehiasarian, J.T. Gudmundsson, Thin Solid Films, 513 (2006) 1-24.

[27] R. Wang, K. Hashimoto, A. Fujishima, M. Chikuni, E. Kojima, A. Kitamura, M. Shimohigoshi, T. Watanabe, Advanced Materials, 10 (1998) 135-138.

[28] A. Fujishima, K. Hashimoto, W. T., TiO2 Photocatalysis: Fundamentals and Applications, BKC, Tokyo, Japan, 1999.

Table I. Summary of deposition conditions and measurements carried out on titania coatings deposited by HiPIMS 


\begin{tabular}{|c|c|c|c|c|c|c|c|}
\hline $\begin{array}{c}\text { Sample } \\
\text { No. }\end{array}$ & $\begin{array}{c}\text { Deposition } \\
\text { pressure, Pa }\end{array}$ & $\begin{array}{c}\text { Pulse } \\
\text { frequency, } \\
\mathrm{Hz}\end{array}$ & $\begin{array}{c}\text { Pulse } \\
\text { width, } \\
\mu \mathrm{s}\end{array}$ & $\begin{array}{c}\mathrm{k}_{\mathrm{a}} \times 10^{5}, \\
\mathrm{~s}^{-1}\end{array}$ & $\begin{array}{c}\text { Water } \\
\text { droplet } \\
\text { contact } \\
\text { angle, }\end{array}$ & $\begin{array}{c}\text { Surface } \\
\text { roughness, } \\
\mu \mathrm{m}\end{array}$ & $\begin{array}{c}\text { Surface } \\
\text { area, } \\
\mu \mathrm{m}^{2}\end{array}$ \\
\hline 1 & 0.16 & 200 & 100 & 1.3 & 82 & 0.0080 & 5525 \\
\hline 2 & 0.33 & 200 & 100 & 2.0 & 63 & 0.0068 & 5521 \\
\hline 3 & 0.49 & 200 & 100 & 2.3 & 59 & 0.0111 & 5520 \\
\hline 4 & 0.66 & 200 & 100 & 2.8 & 26 & 0.0104 & 5520 \\
\hline 5 & 0.93 & 200 & 100 & 3.3 & 9 & 0.0072 & 5520 \\
\hline 6 & 0.33 & 100 & 100 & 1.0 & 64 & 0.0100 & 5519 \\
\hline 7 & 0.33 & 150 & 100 & 1.4 & 64 & 0.0091 & 5521 \\
\hline 8 & 0.33 & 250 & 100 & 1.9 & 52 & 0.0068 & 5525 \\
\hline 9 & 0.33 & 300 & 100 & 2.0 & 34 & 0.0061 & 5523 \\
\hline 10 & 0.33 & 200 & 50 & 2.1 & 73 & 0.0052 & 5520 \\
\hline 11 & 0.33 & 200 & 75 & 1.9 & 69 & 0.0092 & 5521 \\
\hline 12 & 0.33 & 200 & 150 & 1.4 & 37 & 0.0074 & 5522 \\
\hline 13 & 0.33 & 200 & 200 & 0 & 31 & 0.0076 & 5520 \\
\hline
\end{tabular}

\section{List of figure captions}

Figure 1: Schematic overview of the sputtering rig

Figure 2: Voltage, current and power characteristics for the pulse-on phase at $0.33 \mathrm{~Pa}$ working pressure, $200 \mathrm{~Hz}$ frequency and $100 \mu$ s pulse width

Figure 3: Spectrum of UV tubes used for photocatalytic experiments

Figure 4: The results of Raman spectroscopy for titania coatings on glass deposited at various working pressures

Figure 5: First rate order constant value for the process of photodegradation of MB as a function of sputtering parameters (pressure, pulse width, pulse frequency). 
Figure 6: Contact angles of deionised water droplets on the surface of the coating and their dependence on the sputtering parameters (pressure, pulse frequency, pulse width)

Figure 7: First rate order constant value for the process of photodegradation of $\mathrm{MB}$ of the coatings deposited onto various substrate types under optimised conditions

Figure 8: Current characteristics during the pulse-on phase for various settings of working pressure

Figure 9: Current characteristics during the pulse-on phase for various settings of pulse width Figure 10: Current characteristics during the pulse-on phase for various settings of pulse frequency

Figure 1. 


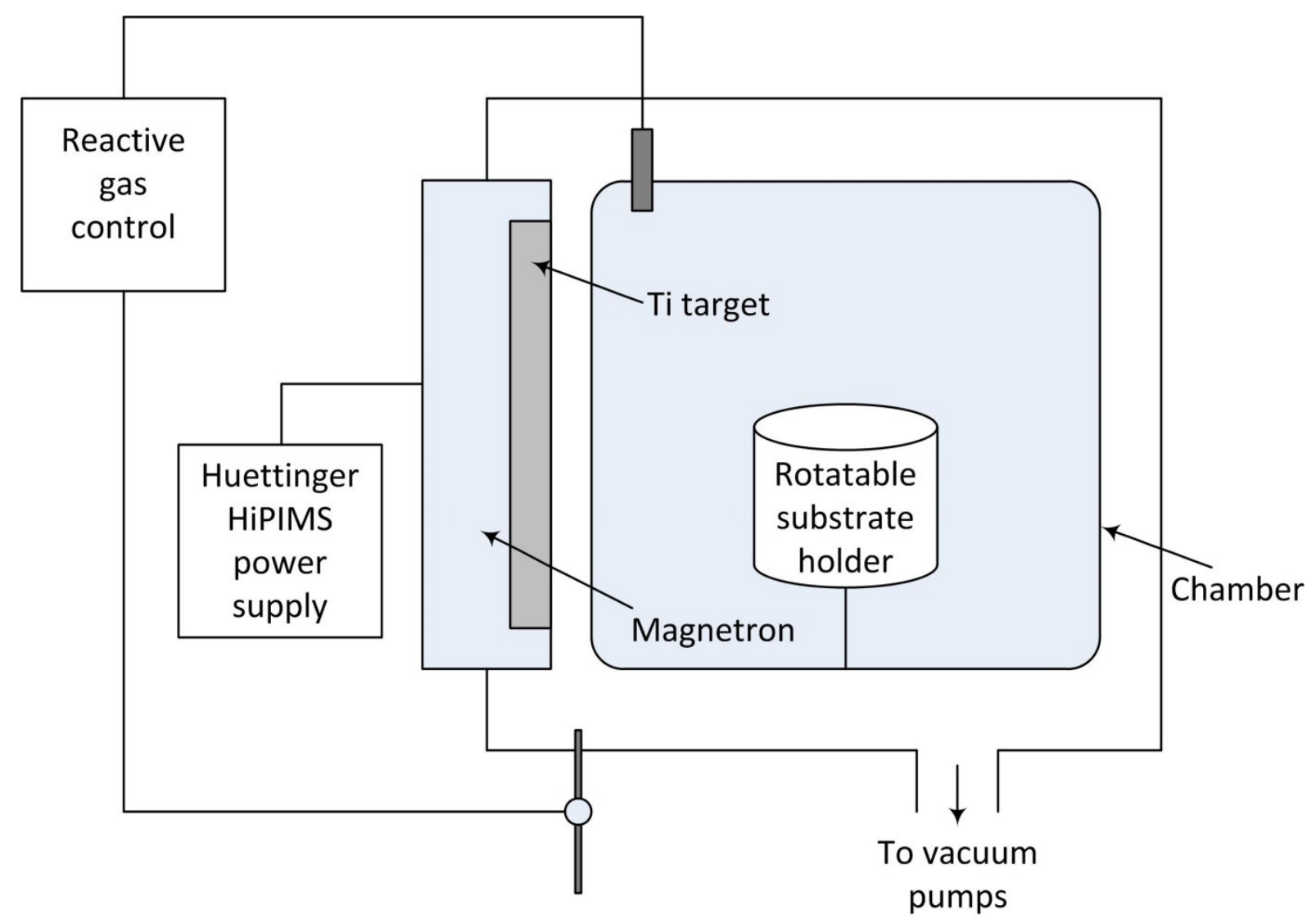

Figure 2 


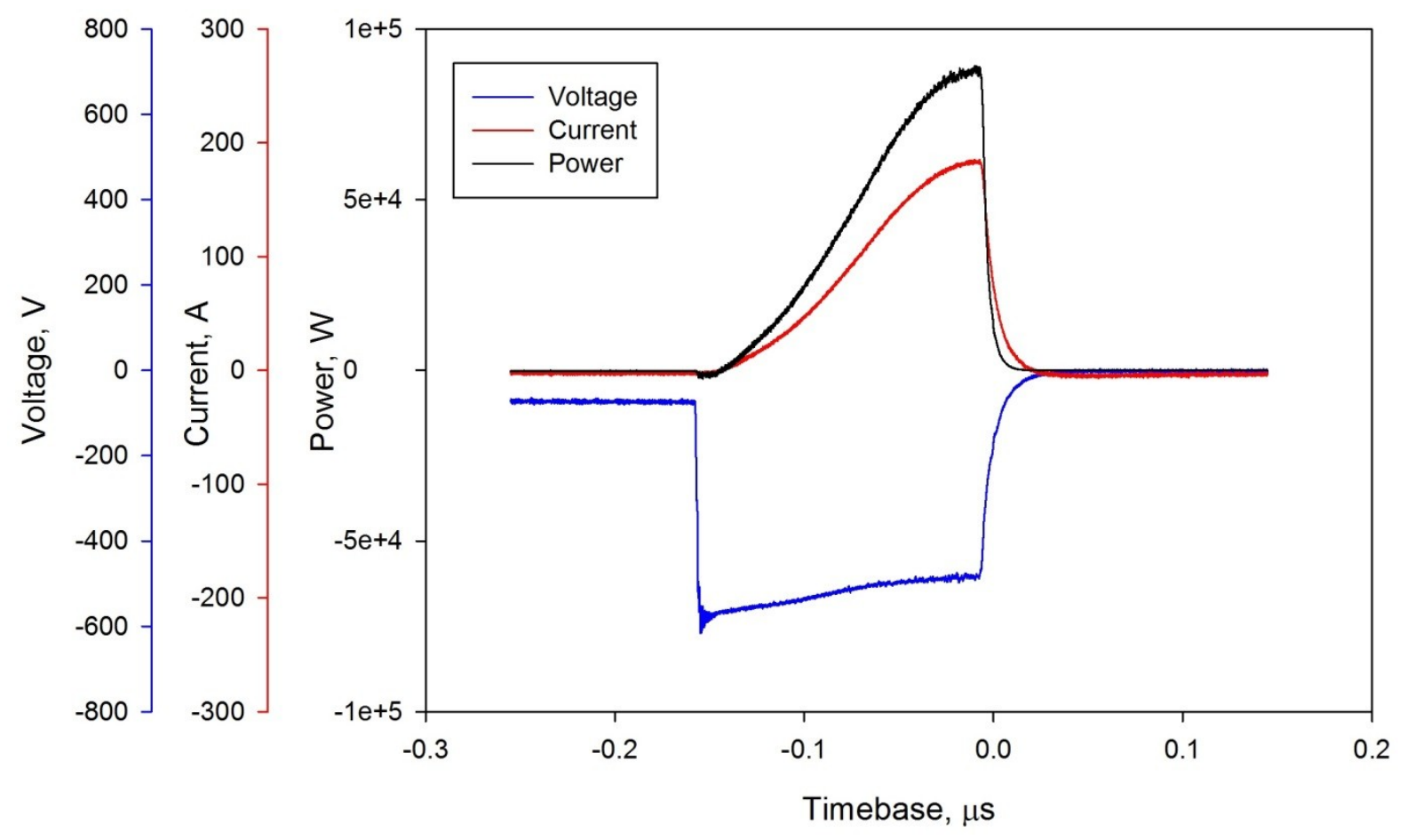

Figure 3 


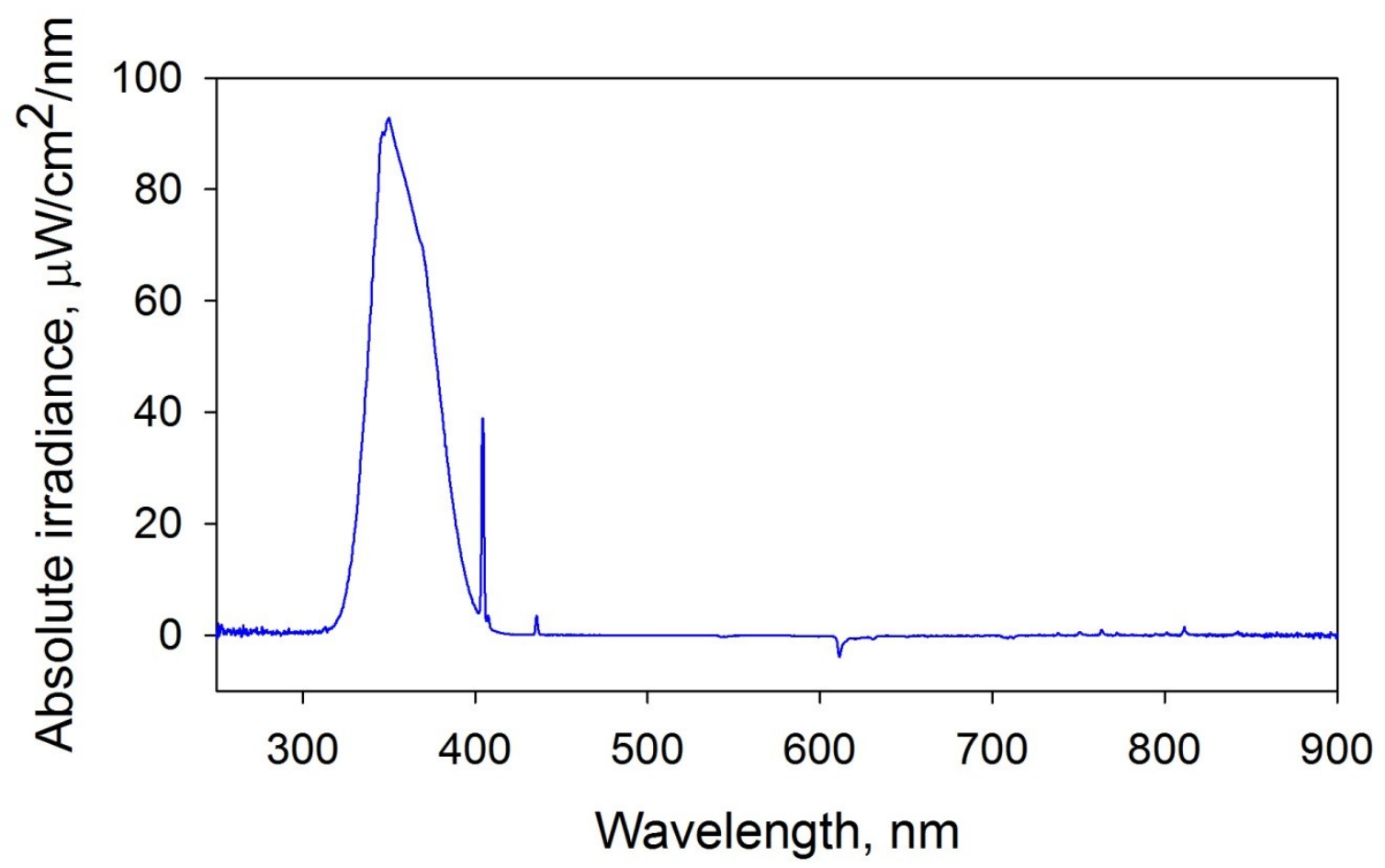

Figure 4 

A
R A R
Ti-O-SiO ${ }_{2}$

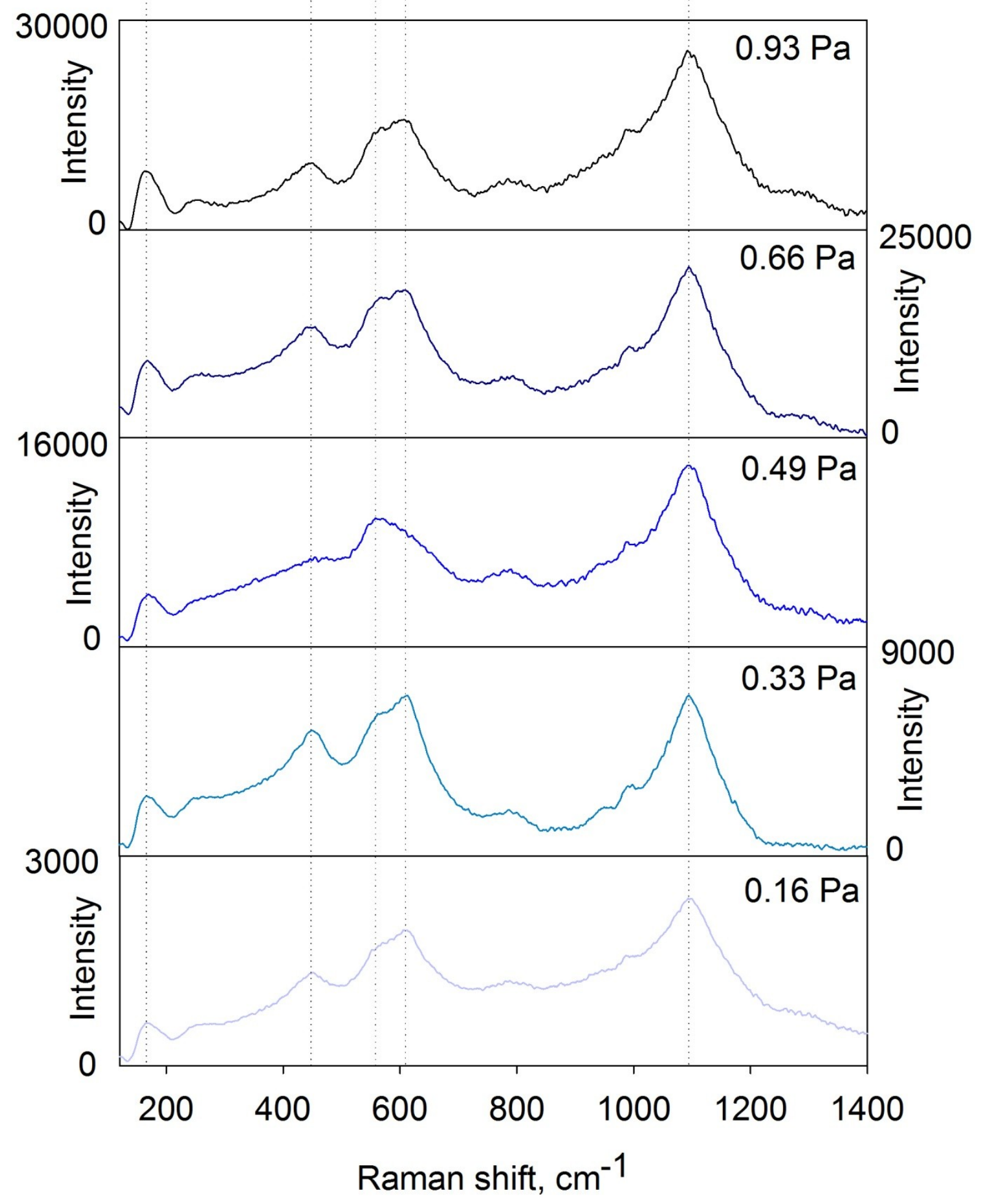

Figure 5 


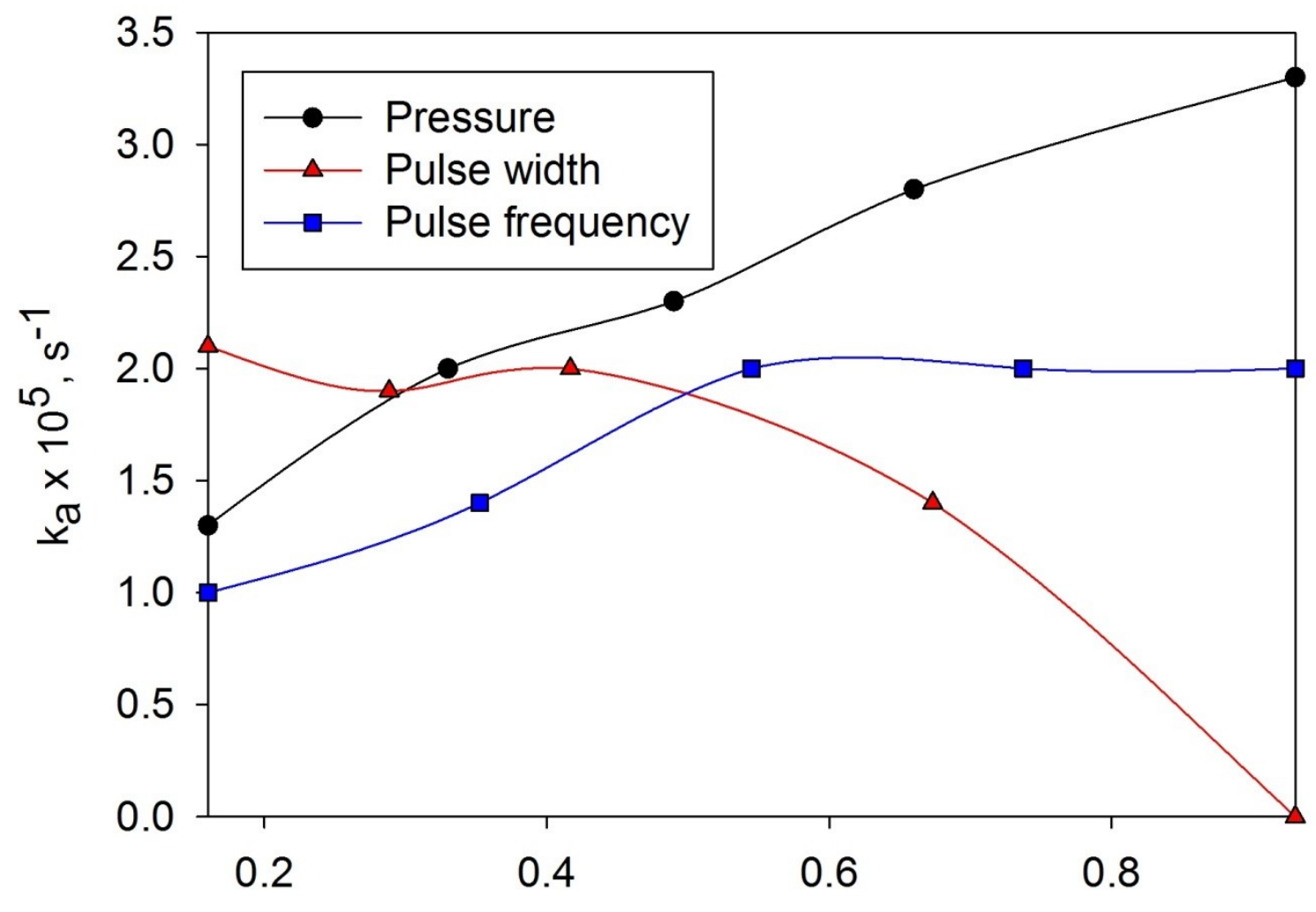

Pressure, $\mathrm{Pa}$

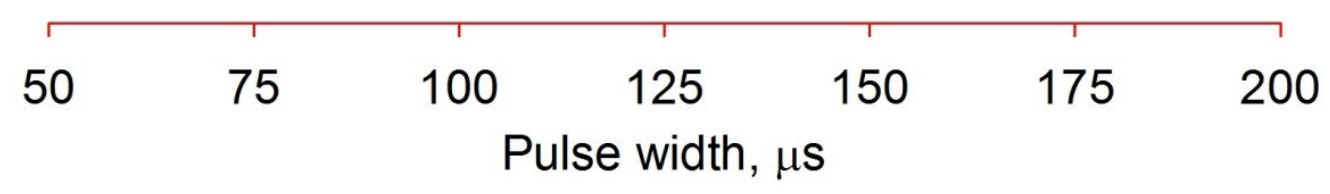

\begin{tabular}{cccc}
\hline 100 & 200 & 250 & 300 \\
& & Pulse frequency. $\mathrm{Hz}$ &
\end{tabular}

Figure 6 


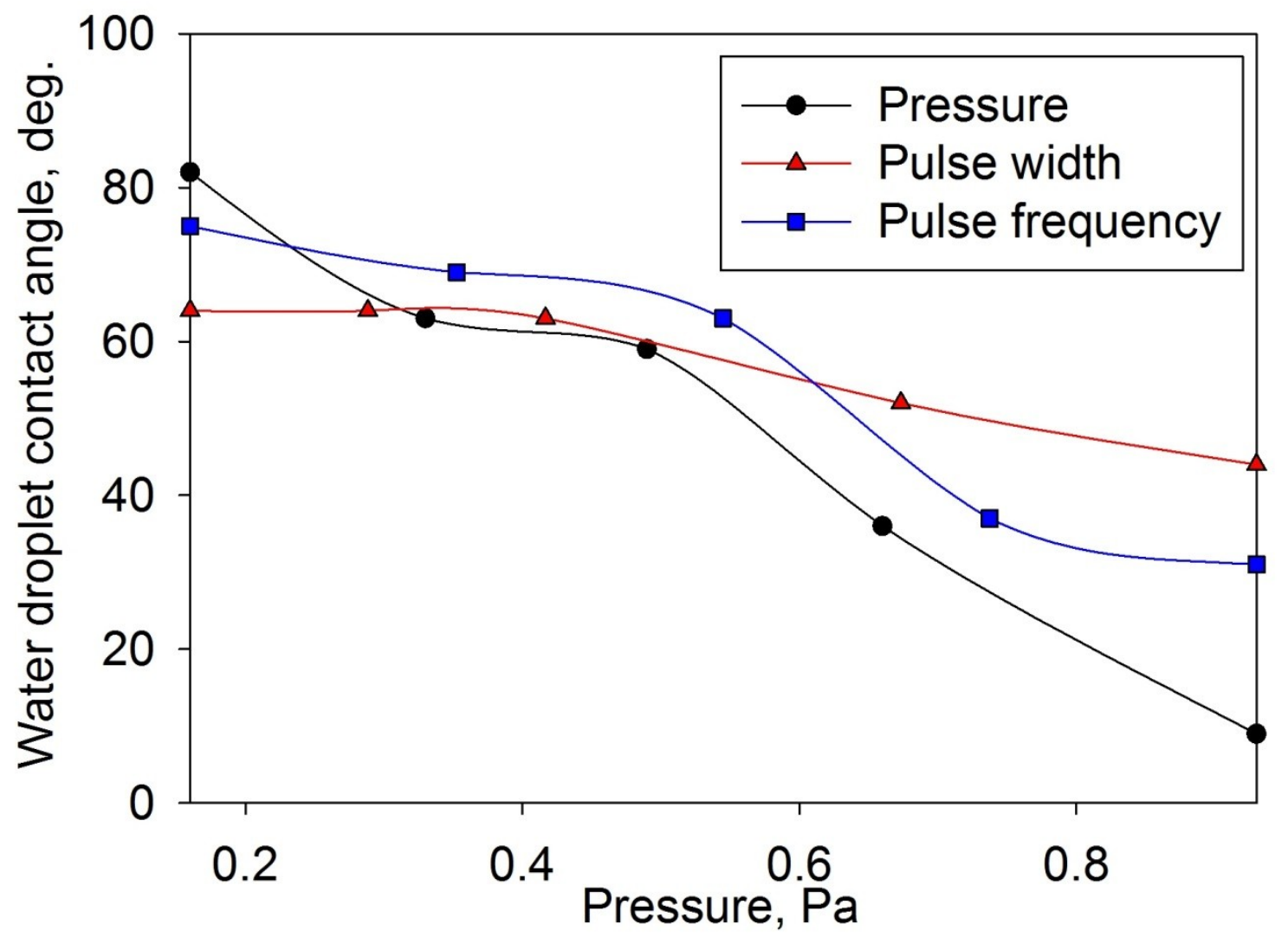

$\begin{array}{cccccc}50 \quad 75 \quad 100 \quad 125 \quad 150 & 175 \quad 200 \\ \text { Pulse width, } \mu \mathrm{s}\end{array}$

$\begin{array}{llll}100 & 150 & 200 & \\ & & & \\ & \text { Pulse frequency. } \mathrm{Hz} & 300\end{array}$

Figure 7 


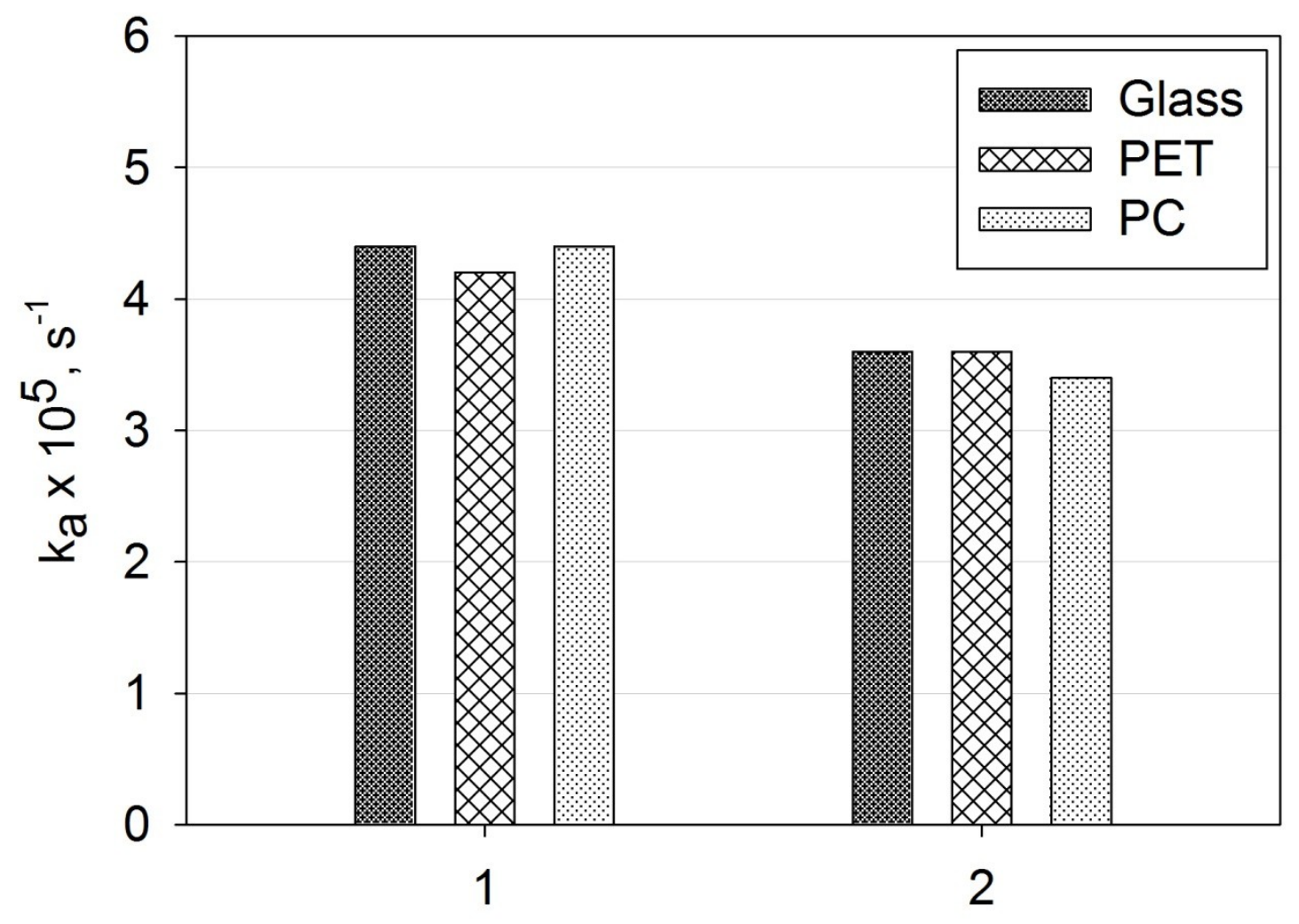

Optimised sputtering conditions set

Figure 8 


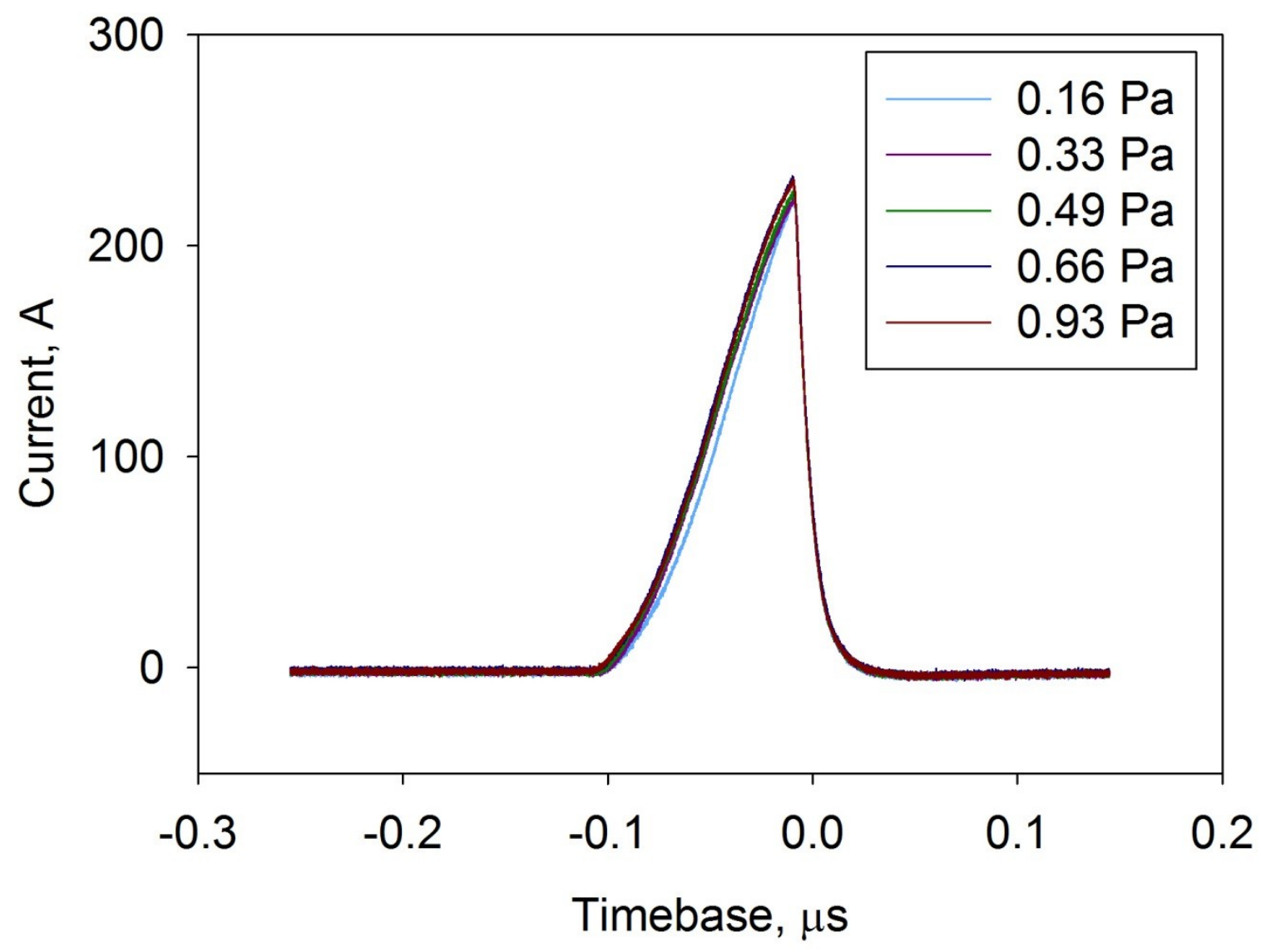

Figure 9 


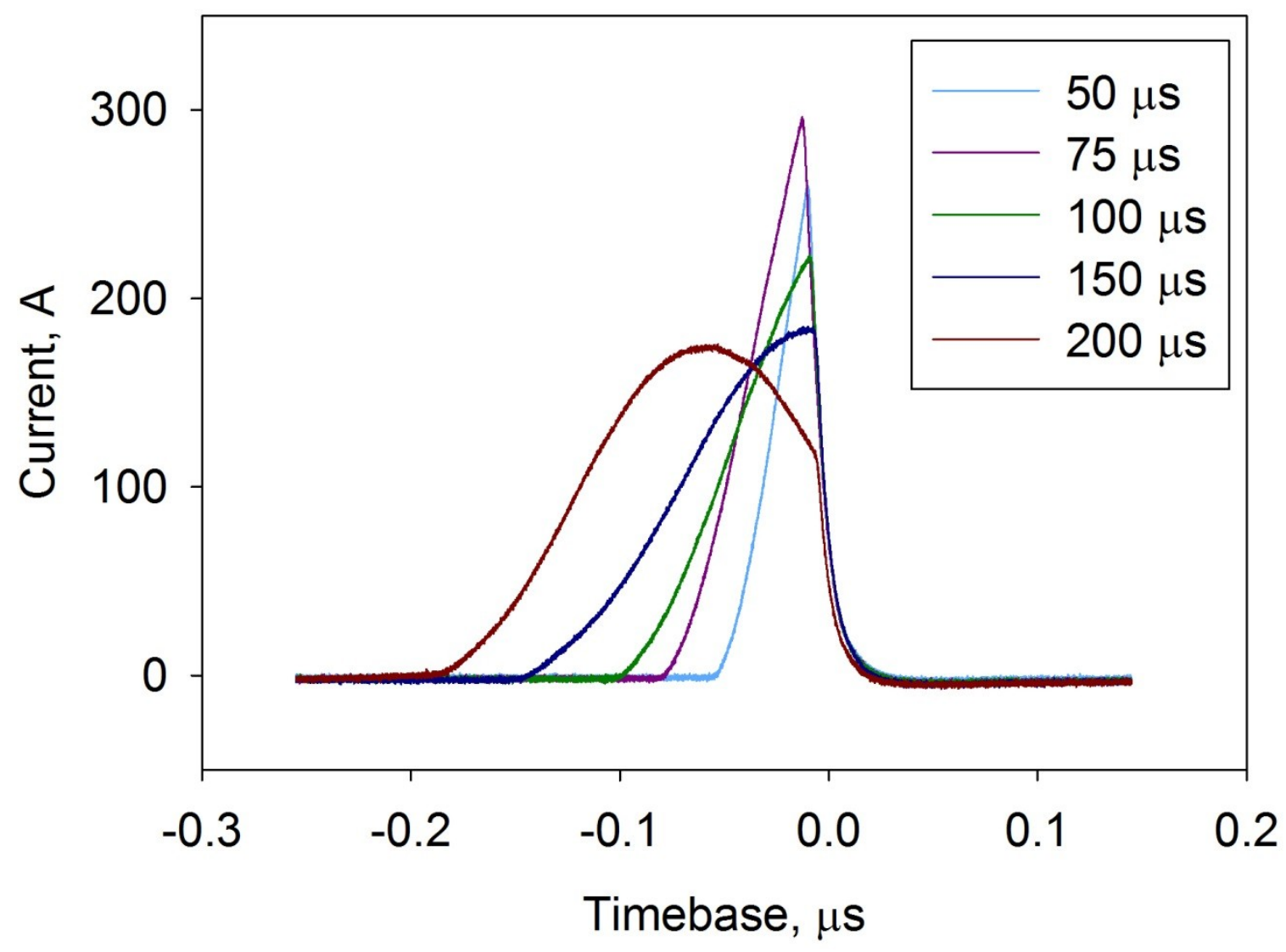

Figure 10 


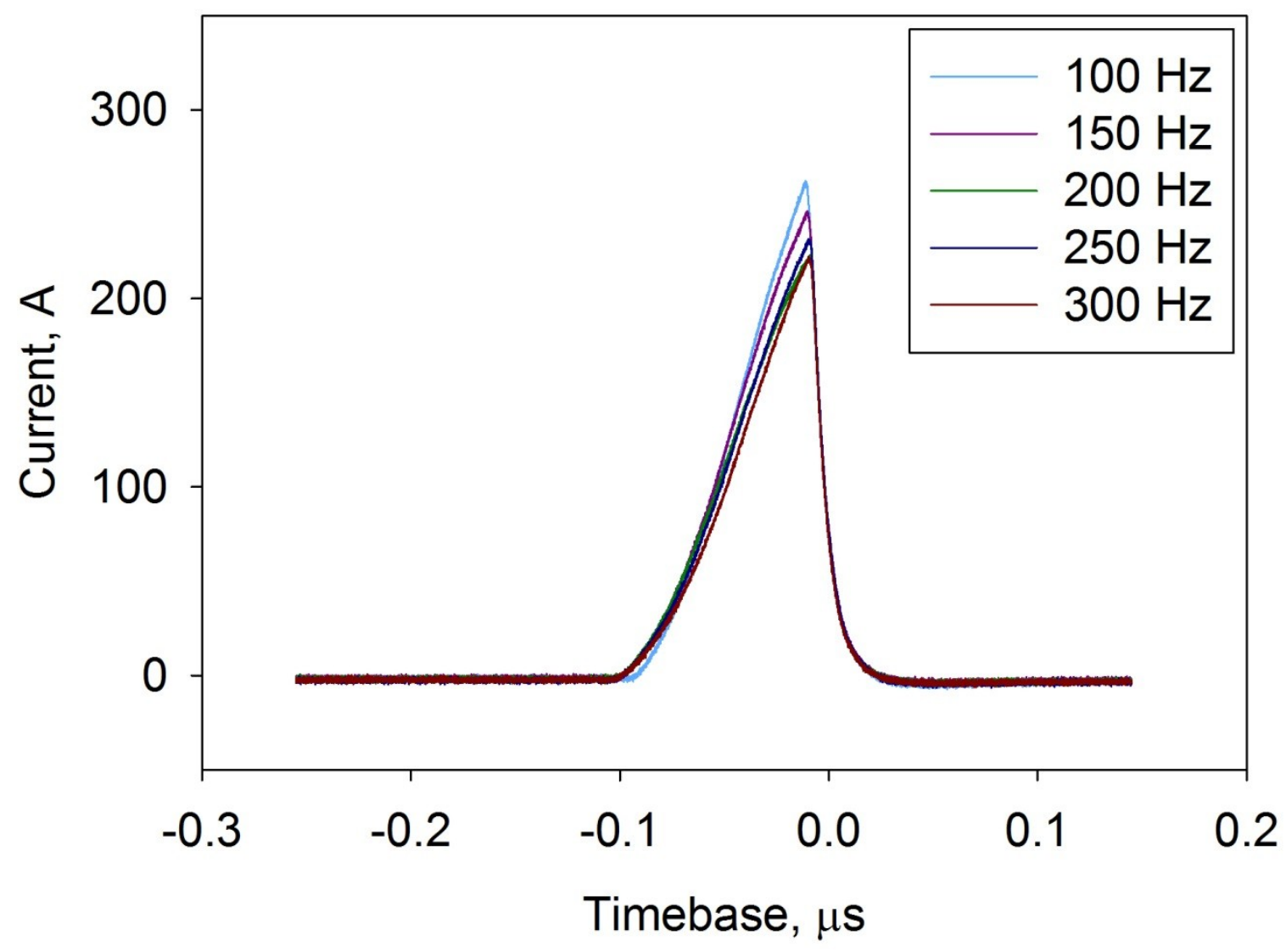

